\title{
BUCHBESPRECHUNGEN
}

Backes, Uwe/Eckhard Jesse/Alexander Gallus (Hg.): Jabrbuch Extremismus \& Demokratie, Band 25

(Harald Bergsdorf)

Hofmann, Hasso: Rechtsphilosophie nach 1945

(Bernd M. Malunat)........................ 373

Kaesler, Dirk: Max Weber - Preuße, Denker, Muttersohn. Eine Biographie

Kaube, Jürgen: Max Weber - Ein Leben zwischen den Epochen

(Hans-Martin Schönherr-Mann)

Richter, Heinz A.: Griechenland 1950-1974: Zwischen Demokratie und Diktatur

(Dirk Schönrock)...

Sörensen, Paul und Nikolai Münch (Hrsg.): $P_{O}-$ litische Theorie und das Denken Heideggers

(Georg Simet).

Sutor, Bernhard: Katholische Soziallebre als politische Ethik

(Bernhard Schreyer)

Wolfrum, Edgar: Rot-Grün an der Macht

(Helge F. Jani). 383

Ilker ATAÇ:, Ökonomische und politische Krisen in der Türkei. Die Neuformierung des peripheren Neoliberalismus, Münster: Westfälisches Dampfboot, 2013, 191 S., 24,90€

Das Buch, die überarbeitete Fassung der Dissertation des Autors zielt darauf ab, »die Geschichte des Neoliberalismus in der Türkei« zu >dekonstruieren (14). Die »Untersuchungsperiode« beginnt daher mit dem dritten Militärputsch in 1980, da in dessen Folge (über die Militärjunta) eine neoliberalistische Tendenz in die bis dahin kemalistischetatistisch verordnete Wirtschaftspolitik des Landes eingeführt wurde. Das Ende des Untersuchungszeitraums ist willkürlich auf den Abschluss der ersten Amtszeit von Erdoğan als Ministerprä- sident 2007 terminiert, dessen Wirtschaftspolitik ebenso wie die der Regierungen vor ihm stark neoliberalistisch geprägt war und ist.

Dieses Zeitintervall von insgesamt 27 Jahren untergliedert Ataç grob in »drei Perioden «/Jahrzehnte: »die 1980er-, und die 1990er- und die 2000er-Jahre«. (15) Diese Grobsubkategorisierung liegt zwar der Gliederung des Buchs zugrunde, doch wird diese Vorgabe dadurch unterlaufen, dass ein zentrales Ereignis das Geschehen dominiert: »Das Programm zum Übergang in eine starke Ökonomie« (Güçlü Ekonomiye Geçiş Programı). Dieses ist zwar erst Gegenstand des sechsten Hauptkapitels, bildet aber gleichwohl den "Mittelpunkt « der Arbeit (13), die folglich diachron nach Veranlassung/»Entstehungshintergrund « (fünftes Hauptkapitel, 15) Ausarbeitung/ "Analyse (sechstes Hauptkapitel) sowie Umsetzung und "Folgen « des Programms (siebtes und letztes Hauptkapitel vor den "Schlussfolgerungen «) gegliedert ist. Der Auseinandersetzung mit diesem Programm vorgelagert sind zunächst ein Kapitel, das die ebenfalls diachron motivierte, »theoretische Herangehensweise " (zweites Hauptkapitel) aufzeigt sowie zwei Kapitel, die daran anschließend die Zeiträume von 1980-88 (drittes Hauptkapitel) und die von 1989-2001 (viertes Hauptkapitel) betrachten. 1980 wird dabei als Beginn und 1989 als »vollständige Öffnung « und "Liberalisierung der inländischen Kapitalmärkte« unterstellt (69). Dieses Konzept der Gliederung ist vom Ansatz her überzeugend, wenn auch die Bedeutung, die dem Jahr 1989 als eine Art Kulminationspunkt im Liberalisierungsprozess zugesprochen wird, nicht deutlich genug herausgearbeitet wird.

Die auf gut 20 Seiten entfaltete Methodik der Darstellung ist vornehmlich durch eine regulations- und eine staatstheoretische Perspektive bestimmt. Grundlage für diese bipolare Ausrichtung ist der radikale Umschwung in der Wirtschaftspolitik von IWF und Weltbank, der als »Paradigmenwechsel im Washington-Konsensus « bezeichnet wird (18). Während ersterem Konzept zufolge beide Organisationen der Auffassung waren, dass »Markt und Staat [...] getrennte Sphären « (19) und »die Mechanismen des Marktes gegenüber dem 
Staat « zu stärken seien, wurde in den 1990er Jahren eine dialektische Position favorisiert, die »eine Betonung der Re-Regulierung des Marktes « zum Ziel hatte (18). Aus regulationstheoretischer Perspektive sind für die weitere Arbeit vor allem zwei $\mathrm{Zu}$ sammenhänge relevant: Das Akkumulationsregime im Allgemeinen und die »Differenzierung zwischen den Ökonomien des Zentrums und Peripherie« im Besonderen (31). Der »Begriff Akkumulationsregime « wird unter Bezug auf Lipietz als »Modus systematischer Verteilung und Reallokation des gesellschaftlichen Produkts « (23) zum Zweck der "produktive[n] Verwertung des Kapitals « verstanden (24). Die»Theorie der Peripherie « erweitert diesen Bezug um die internationale Komponente, die »Integration in die Weltwirtschaft « (31). Staatstheoretisch geht der Autor in Anlehnung an Marx von einer »materialistische[n] Theorie des bürgerlichen Staates « aus (34). Dieser zufolge sind Ökonomie und Staat zwar prinzipiell getrennt, aber aufeinander bezogen. Aufgabe des Staates sei es dabei, die Herrschaft der ökonomisch herrschenden Klasse zu >realisieren $<$ (35). Der Staat fungiere daher lediglich als Mittel zum Zweck der Herrschaftsausübung und -sicherung unter primär ökonomischer Perspektive.

Dieses theoretische Verständnis ist durchaus hilfreich, um die diversen wirtschaftlichen Krisen der Türkei ab den 1980er Jahren in vergleichender Hinsicht zu analysieren. Allerdings gelingt es dem Autor mithilfe dieses Konzepts nicht, die politischen Krisen in ihren nicht-wirtschaftlichen Bezügen und Implikationen adäquat zu erfassen, zu verstehen und zu erklären. Hierzu zwei Beispiele:

Zum einen ist der Aufstieg der AKP kein von den damaligen wirtschaftlichen Eliten inszenierter und gepushter Weg zur Macht, sondern eher ein von ihnen, vom Establishment bekämpfter. Der Aufstieg der AKP ermöglichte es denn auch, neue wirtschaftliche Eliten aus der islamisch-konservativen Wählerschaft in Konkurrenz zu den bestehenden (Holdings von Unternehmerdynastien wie Koç und Sabancı) auszubilden. So zeigt sich, dass politische Vorgänge nicht nur bislang unterdrücktes wirtschaftliches Potenzial freisetzen, sondern hierdurch sogar wirtschaftlichen Aufschwung und extremes wirtschaftliches Wachstum ermöglichen können. Zum anderen ist die Einengung des Krisenbegriffs auf wirtschaftliche Vorgänge problematisch, weil dadurch alle nicht-wirtschaftlich beeinflussten Konflikte und Krisen a priori ausge- schlossen werden. $\mathrm{Zu}$ diesen zählen die religiös und ethisch und primär politisch bedingten. Weder der ethnisch motivierte Kurdenkonflikt, noch der religiös motivierte Brandanschlag von Sivas 1993 und die Ermordung von Hrant Dink 2007, und selbst die machtpolitisch inszenierten Prozesse gegen den »tiefen Staat « können daher vom $\mathrm{Au}$ tor in die Untersuchung einbezogen werden. Der Titel des Buchs, »Ökonomische und politische Krisen in der Türkei «, ist folglich irreführend. Analysiert werden ausschließlich volkswirtschaftliche Vorgänge und Verhältnisse.

Trotz der konzeptionellen Mängel aufgrund eines nicht-dialektischen, einseitigen Verständnisses der Beziehung zwischen Wirtschaft und Politik und eines zu eng gefassten, nämlich auf wirtschaftliche Vorgänge reduzierten Krisenbegriffs ist das Buch jedoch äußerst lesenswert. Denn es gibt einen fundierten Überblick über die wirtschaftliche Entwicklung der Türkei unter Bezug auf die wirtschaftspolitischen Maßnahmen der maßgeblichen politischen Akteure auf nationaler (türkischer) wie internationaler Ebene (IWF und Weltbank), wenn auch aus post-marxistischer Perspektive. Dabei werden insbesondere die "Wirtschaftskrisen von 1994 und 2001 « behandelt (96), um zum einen deren Ursachen und Folgen aufzuzeigen und zum anderen die (Umsetzung der) Konzepte zu ihrer Überwindung auf deren Effektivität hin zu untersuchen. Dass der von Derviş (als Wirtschaftsminister in 2001) initiierte "Strukturwandel von einem >chronisch korrupten System< hin zu einer ,rational funktionierenden Marktökonomie« « allerdings scheiterte (121), zeigt der Ende 2013 aufgedeckte Korruptionsskandal, in den die Regierung und selbst Ministerpräsident Erdoğan verstrickt zu sein scheinen.

Fazit: Das Buch bietet eine detaillierte Darstellung und Analyse der volkswirtschaftlichen Entwicklung der Türkei von 1980 bis 2007. Hierbei werden insbesondere die Ursachen, Folgen und Maßnahmen gegen die ökonomischen Krisen von 1994 und 2001 erörtert. Um die diversen makroökonomischen Entwicklungen besser nachvollziehen zu können, wäre es hilfreich gewesen, die einbezogenen, aussagekräftigen statistischen Daten zumindest im Anhang tabellarisch und/oder graphisch im Überblick darzustellen.

Georg Simet 
Uwe BACKES/Eckhard JESSE/Alexander GALLUS (Hg.): Jahrbuch Extremismus \& Demokratie, Band 25, Baden-Baden: Nomos, 2013, 487 S., $59 €$

Wie in den Vorjahren versammelt auch der 25. Band des Jahrbuchs Extremismus \& Demokratie ein facettenreiches Kompendium an Analysen über verschiedene Extremismen im In- und Ausland. Eckhard Jesse untersucht in seinem Beitrag die empirische Validität der jüngsten Publikationen Elmar Brählers und Oliver Deckers (Rechtsextremismus der Mitte), die seit 2006 alle zwei Jahre im Auftrag der Friedrich-Ebert-Stiftung erforschen, wie verbreitet rechtsextreme Einstellungen in Deutschland sind. Nach Brählers und Deckers aktuellen Zahlen ist in Gesamtdeutschland die Zahl derer, die ein geschlossen rechtsextremes Weltbild in sich tragen, von 8,2 auf $9 \%$ gestiegen - zu einem solchen Weltbild zählen die Autoren Antisemitismus, Chauvinismus, Diktaturbefürwortung, Fremdenfeindlichkeit, Sozialdarwinismus und Verharmlosung des Nationalsozialismus. Stärker als die Vorgängerstudien Deckers und Brählers will die aktuelle Ausgabe ihrer Untersuchungsreihe - bei den Einzelaussagen - differenzieren. So bemüht sie sich, zwischen Islamkritik und Islamfeindlichkeit zu unterscheiden und verschiedene Formen von Antisemitismus zu erfassen - sowohl bei Nichtmigranten als auch bei Migranten.

Tatsächlich sind aber auch in ihren aktuellen Studien viele Fragen sehr unscharf formuliert, wie Jesse moniert. Dadurch verschwimmen Grenzen zwischen Rechtsextremismus und demokratischer Mitte. Kein Wunder ist es deshalb, wenn manche Aussagen auch jenseits des rechtsextremen Potenzials auf zum Teil hohe Zustimmung stoßen. So meinen beide Psychologen und ihr Team, bereits einen Indikator für »Chauvinismus « gefunden zu haben, wenn eine Person dafür plädiert, deutsche Interessen kraftvoll durchzusetzen (Zustimmung: $30 \%)$. Wie viele dieser Befragten befinden zugleich, gerade die europäische Integration diene deutschen Interessen? Gibt es keine Unterschiede zwischen idiotischem Nationalismus und weltoffenem Patriotismus, den keiner teilen muss?

Die Aussage, zuweilen sei eine Diktatur die bessere Staatsform (Zustimmung: $7 \%$ ), dürften auch Linksextremisten unterstützen. Besonders unpräzise formuliert ist die Frage, ob » man « Hitler heute ohne Judenvernichtung als großen Staats- mann ansehen würde (Zustimmung: $11 \%$ ). Fragen die Autoren hier danach, wie Befragte selbst das einschätzen oder wie sie die Einschätzungen ihrer Mitmenschen einschätzen oder beides? Wer der pauschalen Aussage, die islamische Welt sei rückständig und verweigere sich neuen Realitäten, zustimmt, gilt schon als islamfeindlich (Zustimmung: $58 \%$ ). Wie viele junge Demonstranten in islamisch geprägten Ländern oder muslimische Intellektuelle in Deutschland würden dieser Aussage zustimmen?

Lässt sich mit solchen Fragen und Antworten ein ausgeprägter Rechtsextremismus der Mitte belegen, wie Brähler und Decker mit antikapitalistischem Unterton unterstellen? Interessant ist zumindest, wie die Zustimmung sinkt, wenn die Autoren ihre Fragen präziser und weniger suggestiv formulieren. So meinen rund $10 \%$ der Befragten, der Nationalsozialismus habe auch gute Seiten gehabt. $8 \%$ befinden, die Geschichtsschreibung habe die NS-Verbrechen weit übertrieben dargestellt. Rechtsextremismus zu bekämpfen, zählt zu den großen Herausforderungen der Zeit. Dafür braucht es zunächst exakte Diagnosen. Dazu leisten Brähler und Decker keinen übergroßen Beitrag. Nur beiläufig erwähnen sie die überdurchschnittliche Fremdenfeindlichkeit im gesamtdeutschen SPD-Wählerpotenzial, wie Jesse anmerkt.

Uwe Backes untersucht in seinem Beitrag islamfeindlichen Rechtsextremismus. Spätestens seit dem 11. September 2011 mutierte die »Islamisierung « zu einer Obsession vieler Rechtsextremisten, darunter auch solche, die gleichzeitig behaupten, die Massenmorde gründeten auf einer jüdisch-amerikanischen Verschwörung. Der »Kampf « gegen die angebliche »Islamisierung « Deutschlands und Europas gehört zu den Schwerpunkten etwa der rechtsextremen Pro-Gruppierung, die sich gern als harmlose »Bürgerbewegung « inszeniert. Sie meint, im Kampf gegen die vermeintliche »Islamisierung " ein kampagnefähiges Topthema gefunden zu haben, mit dem sie erfolgreicher mobilisieren könne als mit traditionellen Topthemen von Rechtsextremisten. Seit Jahren konzentriert die Pro-Gruppierung ihre Fremdenfeindlichkeit auf Muslime. Um ihr präferiertes Thema in Wahlkämpfen noch anzuschärfen, provozierte die Pro-Gruppierung 2012 in NRW auf Demonstrationen (» Karikaturenwettbewerb «) Salafisten, die bei gewalttätigen Auseinandersetzungen zahlreiche Polizisten zum Teil schwer verletzten. 
Mehrere Merkmale kennzeichnen laut Backes muslimfeindliche Rechtsextremisten in Deutschland und Europa. Zum ersten stilisieren sich viele von ihnen als prozionistisch. Damit wollen sie Vorwürfe aushebeln, sie seien antisemitisch - das ist ein Markenzeichen etwa von Geert Wilders und seiner Ein-Mann-Partei. Zum zweiten qualifizieren sie »den « Islam als »faschistisch «, um sich dadurch als demokratisch zu präsentieren. Drittens gerieren sie sich als Verteidiger des »christlichen Abendlandes «, um als Antinationalisten aufzutreten. Doch die undifferenzierte und hasserfüllte Feindbildpflege solcher Kräfte widerspricht, wie Backes betont, fundamental christlichen Grundwerten wie Barmherzigkeit, Mäßigung, Gerechtigkeit und Nächstenliebe. Langfristig könnten die schrumpfenden Kirchenbindungen und die zum Teil galoppierende Verdunstung des christlichen Glaubens rechtsextremen Kräften zunehmend nutzen - als ein Faktor unter mehreren. Zumindest neigen gerade auch kirchentreue Katholiken bzw. aktive Christen beider Konfessionen in besonders geringem Maße dazu, rechtsextreme Parteien zu wählen (Kenner des NSDAP-Wählerpotenzials meinen gar, in einem rein katholischen Deutschland wäre Hitler nie an die Macht gelangt).

Rechtsextremisten negieren im Kern die Gleichwertigkeit aller Menschen - im krassen Kontrast zur christlichen Botschaft. Sie nennen Muslime fast ausschließlich im negativem Kontext und versuchen, Islam und Islamismus gleichzusetzen, obwohl sich beide beinahe wie Kirchturm und Küchenhocker unterscheiden. Darüber will rechtsextreme Rhetorik hinwegtäuschen. Deshalb warnt sie vor einer "Islamisierung «. Sie will alle Muslime unter Generalverdacht stellen. In Wirklichkeit lebt die übergroße Mehrheit der Muslime gesetzestreu in Deutschland. Sie praktiziert ihren Glauben/ihre Kultur friedlich und will sich integrieren. Nur eine kleine Minderheit der Muslime verbreitet Hass und propagiert bzw. praktiziert Gewalt.

Im Kontrast zur rechtsextremen Agitation gehört das Recht, seinen Glauben friedlich zu praktizieren, essenziell zur freiheitlich-demokratischen Ordnung des Grundgesetzes. Selbstverständlich genießen deshalb auch Muslime in der Bundesrepublik eine garantierte Religionsfreiheit - das heißt Freiheit zur Religion, aber auch Freiheit von Religion. Im Widerspruch dazu gehören zum einen Islamisten zu den Fanatikern, die das fried- liche Zusammenleben in Deutschland gefährden, die (religiösen) Pluralismus und damit letztlich die Freiheit für Nichtmuslime und Muslime ablehnen und abschaffen wollen, indem sie sogar Kinder ermorden (lassen), wobei Muslime weltweit die Mehrheit der Opfer des Islamismus bilden.

Zum anderen weigern sich (islamophobe) Rechtsextremisten, zwischen einer Religion und ihrem politischen Missbrauch zu differenzieren. Doch prägt Ambivalenz das Verhältnis von Rechtsextremisten zum Islam. Einerseits wenden sie sich vehement gegen jegliche Moscheen und damit gegen das Grundrecht auf Religionsfreiheit. Andererseits loben manche Rechtsextremisten den Islam, den sie mit Islamismus gleichsetzen, weil er Integration verhindere, wie sie meinen - Integration gilt unter Rechtsextremisten als Unterwanderung. Letztlich teilen Islamismus und Rechtsextremismus mehrere Ideologiefragmente: Antisemitismus, Antiamerikanismus, Antikapitalismus und NS-Apologetik bzw. -Negationismus sowie vor allem Demokratiefeindlichkeit.

Um (antimuslimische) Rechtsextremisten weiter überdeutlich unter $5 \%$ zu drücken, gilt es, kursierende Klischees zu bekämpfen - bereits Albert Einstein warnte: "Es ist leichter, ein Atom zu spalten als ein Vorurteil.« Tatsächlich beteiligt sich entgegen vielen Annahmen oder Behauptungen die übergroße Mehrzahl der Schüler und Schülerinnen aus muslimisch geprägten Ländern (darunter irakische Christen) sowohl am Schwimmunterricht als auch an Klassenfahrten. Ebenfalls abwegig ist es, »den « Islam in seiner Vielgestaltigkeit auf sogenannte "Ehrenmorde " und "Zwangsheiraten « $z u$ reduzieren, die natürlich ebenso unmissverständlich zu verurteilen und zu bekämpfen sind wie Islamismus und Integrationsverweigerung als solche, etwa von muslimisch geprägten Jugendlichen, die muslimisch geprägte Mädchen zwingen (wollen), Kopftuch zu tragen. Umso wichtiger ist es, Integration sowohl konsequent zu fordern als auch zu fördern - gerade auch im Interesse und im Sinne der vielen gut integrierten Migranten in Deutschland.

Wie stets verbinden die Herausgeber klare Positionierungen mit einem wachen Interesse für andere Auffassungen. Das zeigt u.a. die Rubrik »Kontrovers besprochen «, in der vier Rezensionen - aus sehr unterschiedlicher Perspektive - ein aktuelles Buch über Vergangenheitspolitik beleuchten. Wiederum fungiert das Jahrbuch als Seismo- 
graf und Kompass für Debatten über Extremismus und Demokratie im In- und Ausland. Auch sein 25. Band stiftet tief schürfende Orientierung. Erneut liefert das Jahrbuch viel sachliche und sachkundige Information, viel multiperspektivische Aufklärung, viel fundierten Diskussionsstoff - für Wissenschaftler, Journalisten, Lehrer und weitere Interessierte/Experten.

\section{Harald Bergsdorf}

Hasso HOFMANN: Rechtsphilosophie nach 1945. Zur Geistesgeschichte der Bundesrepublik Deutschland, Berlin: Duncker \& Humblot, 2012, 75 S., $18 €$

Es ist ein anspruchsvolles Unterfangen, die Geistesgeschichte der Bundesrepublik Deutschland nach $1945 \mathrm{im}$ Lichte der Rechtsphilosophie zu betrachten. In einer kleinen Schrift, die auf einem Vortrag bei der Carl Friedrich von Siemens Stiftung in München basiert, skizziert der renommierte Rechtsphilosoph und Verfassungsrechtler die für ihn entscheidenden Orientierungen, die den Weg der Republik bestimmten und teils weiterhin bestimmen.

Hofmann vermeidet es, die verschiedenen rechtsphilosophischen Strömungen der jeweiligen Zeiten nachzuzeichnen. Stattdessen orientiert er sich an den wechselnden Entwicklungsphasen des rechtsphilosophischen Denkens im Rahmen und als Teil des allgemeinen politischen, wirtschaftlich-sozialen sowie geistig-kulturellen Wandels, als einem mehr oder minder ganzheitlichen Prozess also.

Die Lehrtraditionen der rechtsphilosophischen Schulen der Weimarer Zeit nach dem perversen Kulturbruch des NS-Regimes umstandslos aufzugreifen, verbot sich wie von selbst. Der desavouierte Rechtspositivismus erzwang in den Jahren der konservativen Konsolidierung der westdeutschen Gesellschaft nach 1945 gewissermaßen eine Renaissance des - christlichen wie existentialistischen - Naturrechts. Diese Art von >Bewältigungsliteratur < verlor - wie die Vorstellungen der katholischen Soziallehre von der sozialen Verantwortung des Eigentums - mit dem langsam einsetzenden >Wirtschaftswunder< Bedeutung und Einfluss. >Aufgehoben< wurde das Naturrechtsdenken jedoch erst durch die Judikatur des Bundesverfassungsgerichtes, das in den Grundrechten der Verfassung eine >objektive Wertordnung< erkannte, womit die Bundesrepublik durch die »Wiedergeburt der Rechtsordnung aus dem Geist der Grundrechte (eigentlich) erst aus der Taufe gehoben wurde«, so der Rechtswissenschaftler Gerd Roellecke. Der Verfassungsentwurf von 1949 ist damit gewissermaßen Naturrecht geworden.

Gesellschaftlich, politisch und auch juristisch folgte auf diese »Grundlegung « eine Welle des »Umbaus«, mit vielfältigen Modernisierungen des Rechtsdenkens, die unter dem Motto »Rechtstheorie statt Rechtsphilosophie«standen. Vor dem gesellschaftlichen Hintergrund dieser »Reformzeit « mit ihren euphorischen Erwartungen an eine planvolle Modernisierung, entwickelten sich vielfältige Diskurse über Verständnis, Anwendung und damit auch Gültigkeit von Recht, die sich angesichts ihrer Komplexität in Kürze aber kaum angemessen darstellen lassen.

Nach dieser Phase theoretischer Analysen des Rechts kehrt mit Beginn der Krise des Sozialstaates die praktische Philosophie in den Mittelpunkt der Erörterungen zurück. Im Zentrum steht dabei der Begriff der Gerechtigkeit, insbesondere der sozialen Gerechtigkeit. Hofmann beschränkt sich dabei allerdings auf eine Diskussion von Rawls Theorie der Gerechtigkeit und deren Schwächen, die er vor allem an der Argumentation von Habermas und Kesting verdeutlicht.

In einem ganz kurzen Schlusskapitel beschäftigt sich Hofmann unter der Chiffre 'Globalisierung< dann mit der "Ankunft in der Weltgesellschaft «. Allerdings begnügt er sich mit Erörterungen über Kants Schrift Zum ewigen Frieden, ohne auf die prinzipiell veränderte Rolle der vereinten deutschen Staaten einzugehen, und auch die jüngsten rechtsphilosophischen Herausforderungen, die sich aus der Umwelt-, der Migrationsproblematik oder auch im Zusammenhang mit den neuen Informationstechnologien ergeben, bleiben ausgeblendet.

Die kleine aber sehr gehaltvolle Schrift wendet sich in den Eingangs-Kapiteln vornehmlich an Verfassungs- und Staatsrechtler, gegen Ende aber auch an allgmein politisch interessierte Leser und darf all jenen zur Lektüre empfohlen werden, die auf engem Ram eine Orientierung über den geistig-rechtlichen Rahmen der bundesdeutschen Nachkriegsgeschichte suchen.

Bernd M. Malunat 
Dirk KAESLER, Max Weber-Preuße, Denker, Muttersohn. Eine Biographie, München: C.H. Beck, 2014, 1007 S., $38 €$ und

Jürgen KAUBE, Max Weber-Ein Leben zwischen den Epochen, Berlin: Rowoblt, 2014, 495 S., $€ 26,95 €$

Wer war Max Weber? Joseph Schumpeter - so der emeritierte Marburger Soziologe Dirk Kaesler in seiner zum 150. Geburtstag am 21. April erschienenen Biographie - bezeichnet Max Weber 1920 in seinem Nachruf als "Schwanenritter mit der silbernen moralischen Rüstung «, als »Gestalter und Beherrscher von geistigen Strömungen «. »Er war nicht konventionell. Er war nicht zugeritten. Er gehörte sich selbst. Aber das ist nicht alles. (...) Er imponierte. Man fügte sich ihm, ob man wollte oder nicht. Kraft klang in jedem seiner Worte, strömte sozusagen aus allen Poren seines Wesens. Kraft in allen Spielarten (..) gleichzeitig intellektuelle und moralische.«(774)

$\mathrm{Ob}$ er sich wirklich selbst gehörte, ob er sich selbst hinlänglich regierte, das darf allerdings bezweifelt werden, nicht nur weil er jahrelang an Depressionen und Nervenzusammenbrüchen litt. Auch nach seiner fragilen Genesung befürchtete er ständig Rückfälle, litt an Schlafstörungen und konsumierte daher Unmengen von Medikamenten.

Mangelnde Selbstbeherrschung Webers in fast allen Lebensbereichen lassen sich mit Jürgen Kaube konstatieren, der in seiner ebenfalls neu erschienenen Weber-Biographie schreibt: »Nervös, ungeduldig, unbeherrscht, abrupt, extrem reizbar, speziell auch in der intellektuellen Sphäre, teils verbohrt in, teils angeekelt von Monotonie und in pausenlose Arbeit mehr flüchtend als ihr gelassener Herr. (...) Im März 1894 schreibt er an die Gattin aus Posten, wo er stationiert ist, dass man dort als meine einzige ins Gewicht fallende Eigenschaft meine Konsumfähigkeit in Bezug auf alkoholische Getränke schätzt und schätzt<. (...) Die Gattin ihrerseits schreibt an die Schwiegermutter im Februar 1895 aus Freiburg: >Max hat sich natürlich noch viel mehr als ich geödet - und behauptet, mindestens 40 Butterbrote aus Rache und etwa 20 Bier vertilgt zu haben, so dass er sich hernach einer boa constrictor ähnlich fühlte<, zumal er sich noch sechs Berliner Pfannkuchen einverleibt habe.« (118f.)

Max Weber füllte Hörsäle. Er hatte ein solches Renommee, dass ihn der deutsche Außenminister
1919 nach Versailles zu den Friedensverhandlungen nach dem ersten Weltkrieg mitnahm. Aber seine spätere eminente wissenschaftliche Bedeutung konnte er selber nicht absehen. »Gegen Ende seines Lebens « - so Jürgen Kaube - »fragt ihn seine Geliebte Else Jaffé, ob er jemals einen Menschen habe sterben sehen. Er verneint. Und sie daraufhin: 'Tod nicht, Geburt nicht, Krieg nicht, Macht nicht - so als ob das Schicksal einen Schleier zwischen ihn und die Realität der Dinge gebreitet habe, $-o b$ das vielleicht sein «Stern> sei? Und er, so ein paar Worte vor sich hinflüsternd, - ja, es sei wohl so. (...) keine Kinder, kein Krieg, kein Vermögen, kein Einfluss: Dass ein Autor, ein Gelehrter oder ein Künstler zum Zeitpunkt seines Todes oder kurz danach völlig gescheitert scheint, ist nicht so selten. Seltener schon, wenn er an diesem Nullpunkt seiner eigenen Ambitionen und der Erwartungen, die an ihn gerichtet waren, aus der großen Höhe einer gesicherten Existenz und eines vielversprechenden Anfangs angekommen ist." (26)

Selbst seine Theorien, die ihn posthum berühmt machen, verblassten. Die Soziologie, die Weber mitbegründete, hat sich längst weiterentwickelt. Seine Wissenschaftslehre, mit der er philosophisch Recht behalten wird, antizipiert zwar die avanciertesten Sprachphilosophien von Ludwig Wittgenstein, Martin Heidegger und Jean-Francois Lyotard. Die spätere Wissenschaftstheorie wird ihn weitgehend missachten, nicht zuletzt, weil er ähnlich wie Nietzsche daran zweifelte, dass man die Wirklichkeit mit wissenschaftlichen Begriffen adäquat abbilden könnte. Von einer solchen Schwäche hören die Verfechter einer objektiven Vernunft nicht gerne, noch dazu, wenn Weber parallel den Vernunftfortschritt als ambivalenten Rationalisierungsprozess begreift.

Die Theorie, mit der er zu Lebzeiten schon recht bekannt wurde, dass der Kapitalismus durch die Ethik des asketischen Protestantismus - hauptsächlich von Calvinisten und Puritanern - entscheidend angetrieben wurde, diese Theorie wird bis heute immer wieder in Frage gestellt. Dass er diesen Zusammenhang bei seinen Romaufenthalten während seiner jahrelangen Krankheit vor 1904 entwickelte, hält Jürgen Kaube für ein besonderes Paradox, das im Zentrum seiner WeberBiographie steht. Denn es drängte sich im Angesicht des Petersdomes die folgende Frage auf: »Wie konnten >Prunk und göttliche Verwaltung eigentlich aus einer in ihren Anfängen weltablehnenden 
Religion, dem Christentum entstehen? (...) Die Antwort auf die Frage (...) lautete: durch ungeplante Nebeneffekte. (...) Was die Zeitgenossen als Folge göttlichen Segens der Mönche interpretiert hätten, sei letztlich rim stärksten Maße Folge ihrer rationalen Wirtschaft gewesen. (...) Doch was er dann schrieb, war keine Geschichte der paradoxen Erfolge des Mönchstums (...) >Seine These läuft darauf hinaus, dass der Protestantismus den Katholizismus an paradoxen Nebenfolgen noch übertroffen und die eigentliche Nachfolge des christlichen Mönchstums angetreten hat.«(143)

Kaube führt damit indirekt vor, dass Weber wie Nietzsche genealogisch denkt. Nietzsches Genealogie will nicht nur kausale Erklärungen meiden und Komplexität nicht reduzieren, sondern ihr gerecht werden. Vor allem aber leitet sie eine Angelegenheit nicht aus deren Geschichte ab, sondern aus ihrem Gegenteil und aus Gegensätzen, die zur Angelegenheit selbst gar nicht recht passen wollen, so Nietzsche die Moral aus dem Willen zur Macht bzw. das Gute aus dem Bösen. Dementsprechend sieht Weber in der Weltlichkeit einen Nebeneffekt der Weltablehnung, die speziell bei den Protestanten zu Weltbeherrschung führt, wie deren Askese zum Reichtum.

Auch psychologisch lässt sich hier eine Parallele zu Nietzsche zeichnen. Ähnlich wie Nietzsche, der ständig kränkelnd die Theorie der Stärke, des Willens zur Macht entwirft, bewundert Weber die protestantische Askese, der er im eigenen Leben gar nicht genügen kann. Nur in seiner Ehe mit seiner Frau Marianne pflegt er in Sachen Erotik eine gehörige Portion Askese. Ja, er propagiert die Askese als Prinzip der Ehe. So schreibt er in seinem Bewerbungsschreiben an seine Zukünftige: »Keine phantasievolle Hingabe an unklare und mystische Seelenstimmungen dürfen wir in uns dulden. Denn wenn die Empfindung Dir hoch geht, musst Du sie bändigen, um mit nüchternem Sinn Dich steuern zu können.«(zit. 95) Er selbst hätte ebendies bitter nötig gehabt.

Schwerlich gelang ihm das in anderen ehelichen Lebensbereichen als der Sexualität. Und nicht mal in dieser Hinsicht hält er den Asketismus in seinem Leben durch. Er pflegte in den letzten Jahrzehnten Beziehungen zu zwei anderen Frauen und zwar mit Zustimmung von Marianne Weber, die ihrerseits offenbar, seine Worte in die Tat umsetzend, einen hochrationalen Umgang mit ihrer Ehe pflegte. Deswegen, nicht nur weil sie auch als Autorin feministischer Bücher in Erscheinung trat, könnte man sie als Vorläuferin der Beziehung von Simone de Beauvoir und Sartre bezeichnen.

Als Weber wegen Geldmangels und auch um in der Nähe von Else Jaffé zu sein, 1919 einen Ruf nach München annahm, geriet er in die politischen Wirren in Bayern, was allerdings nur dazu führte, dass er sich in seinen politischen Theorien umso mehr verschanzte, die heute zwar immer noch fleißig gelesen werden. Doch geschätzt werden sie höchstens von jenen, die eine sogenannte Realpolitik fordern und von einem autoritären Machtstaat oder wenigstens einer gelenkten Demokratie träumen.

Denn Demokratie schätzte Weber nur als eine effiziente Form der Führerauslese. Nicht nur entwickelte er dementsprechend den Begriff des charismatischen Führers. Als Mitglied der verfassungsgebenden Versammlung in Weimar plädierte er für die Direktwahl eines mit Machtfülle ausgestatteten Präsidenten, der später in der Person von Hindenburg Hitler den Weg bereiten sollte. Nach Kaesler berichtet Marianne Weber über eine Begegnung ihres Mannes mit Erich Ludendorff, dem Erfinder der Dolchstoßlegende, der Weber fragte: »Was verstehen Sie dann unter Demokratie? Weber: In der Demokratie wählt das Volk seinen Führer, dem es vertraut. Dann sagt der Gewählte: >Nun haltet den Mund und pariert.< Volk und Parteien dürfen ihm nicht mehr hineinreden. Ludendorff: Solche >Demokratie kann mir gefallen!« (885)

Bereits 1895 forderte Weber in seiner berühmt gewordenen Freiburger Antrittsrede aus liberaler Perspektive einen deutschen Imperialismus, wobei es, wie Kaube betont, darum ging, dass der Nutznießer einer Kolonialpolitik das Bürgertum sein würde, was auch dessen politische Macht gegenüber dem Adel stärke. Dadurch würde dann auch innenpolitisch langsam mehr Liberalität einziehen, was aber keinesfalls politische Partizipation der Arbeiter bedeuten sollte, gar ein Ende des Untertanen, den Weber noch in seinem Vortrag Politik als Beruf 1919 beschwören wird.

Aber nicht nur dass dieser deutsche Imperialismus de facto den Weg in den Ersten Weltkrieg ebnete. Weber war Nationalist, war einige Jahre Mitglied im radikalen Alldeutschen Verband, den der Hitlersteigbügelhalter Hugenberg gegründet hatte, kämpfte für die Reinheit des Deutschtums und forderte deutsche Werte für die Wissenschaft, was 
besonders paradox ist, da er ja andererseits für die Werturteilsfreiheit in den Wissenschaften plädierte. Dirk Kaesler hat diese dunkle Seite Max Webers in seiner Biographie besonders hervorgehoben. "Die Parolen von der Gefährdung des >Deutschtums $<$ durch die unkontrollierten Einwanderungswellen der Wanderarbeiter vornehmlich aus Russisch-Polen und Galizien, in ihrer heute nur schwer erträglichen Kombination von xenophoben und antisemitischen Tönen mit GroßmachtPhantasien, erinnern fatal an spätere und gegenwärtige Agitationen gegen die ,Überfremdung, Deutschlands.«(408)

Noch unerfreulicher erscheint Webers Kriegsbegeisterung nicht nur am Beginn des ersten Weltkriegs. Dirk Kaesler zitiert aus einem Brief Webers vom 15. Oktober 1914: »Dieser Krieg ist bei aller Scheußlichkeit doch groß und wunderbar, es lohnt sich ihn zu erleben - noch mehr würde es sich lohnen, dabei zu sein, aber leider kann man mich im Feld nicht brauchen, wie es gewesen wäre, wenn er rechtzeitig - vor 25 Jahren - geführt worden wäre.« (739) Offenbar war Max Weber schon Jahrzehnte lang nicht für den Frieden, sondern für den Krieg. Im September 1917 preist er den Kauf von Kriegsanleihen als gute Geldanlage und als nationale Pflicht. Nicht nur er selbst verlor damit einen großen Teil von den Resten seines ererbten Vermögens, so dass er sich wieder um eine feste Professur bemühen musste. Er ruinierte auch seine Mutter, die er zum Kauf motivierte.

Als er daraufhin im letzten Kriegssommer 1918 einen Lehrstuhl in Wien vertrat, war das Hauptgebäude der Universität, in dem er Lehrveranstaltungen abhielt, teilweise ein Lazarett für Verwundete. Dirk Kaesler schreibt dazu: »Es ist unmöglich, dass Max Weber diesen finsteren Anblick auf dem Weg in seine Vorlesung im Hauptgebäude vermeiden konnte, in dem es gewiss nach Tod und Verwesung gestunken hat und durch das die Schreie und das Stöhnen der verletzten Soldaten zu hören gewesen sein müssen. Dennoch findet sich keine Erwähnung davon in der uns erhaltenen Korrespondenz.«

Auch Jürgen Kaube kritisiert, dass Weber seinen kühlen soziologischen Blick aufgibt, wenn es um seine eigenen letzten und höchsten Werte geht: »Im Oktober >1914< ist der Krieg (...) ein sheiliger Verteidigungskrieg< und der Geist der Truppen ,von strahlender Herrlichkeit‘.« (350) Als 1915 sein Bruder Karl im Russlandkrieg umkommt, spricht er der Mutter Trost zu mit den Worten: »er fand den schönen Tod an der Stelle, wo es im Augenblick allein menschenwürdig ist zu stehen.« (351) Kaube kommentiert das mit den Worten: »Im Begriff der shistorischen Pflicht< zum Krieg geht das soziologische Licht aus.«(352)

Kaubes Biographie, halb so umfangreich wie diejenige Kaeslers, ist spannend geschrieben, gut lesbar und besitzt insoweit auch literarische Qualitäten, als sie ein Professorenleben lebendig macht, das ja strukturell eher zur Routine und somit zur Langeweile neigt. Aber für Weber - so Kaube - sind die innerweltlichen Asketen, die allerdings anders als er selbst zur methodischen Lebensführung fähig sind, die wahren Helden.

Kaeslers Biographie wirkt demgegenüber manchmal schwerfällig, nicht zuletzt, da sie ungeheuer viel und lang zitiert. Doch diese Zitate belegen zumeist nicht nur seine Thesen, sondern erhellen dieses Professorenleben nachhaltig, unter anderem mit einem Tagebucheintrag des Historikers Karl Hampe über einen Vortrag Max Webers nach dem Friedensvertrag von Versailles, bei dem Weber feststellte: »Das A und O jeder Politik müsse die Abänderung des Friedensvertrages sein, jede Handlung müsse sich nach diesem Ziel richten. Die Zeit dazu sei noch nicht gekommen.«(888)

Max Weber hat also sowohl objektiv historisch durch sein Plädoyer für die Machtfülle und Direktwahl des Präsidenten der Weimarer Republik, als auch gedanklich in vielfältiger Hinsicht Hitler den Weg bereitet: Die Nazi-Ideologie stützte sich unmittelbar auf das imperiale, alldeutsche und bellizistische Denken, wie es auch Carl Schmitt vertritt: Der Normalzustand ist der Ausnahmezustand und Demokratie höchstens Akklamation; alles hat sich dem Krieg unterzuordnen; der Bürger ist höchstens Untertan.

Das ändert natürlich nichts an der Relevanz von Max Webers Religionssoziologie und seiner Wissenschaftslehre, taucht höchstens seine politische Theorie in ein fragwürdiges Licht, das damit auch auf viele andere fällt, die Politik auf Gewalt gründen. Ein Vergleich zu Martin Heidegger drängt sich auf: Philosophen, die avancierte Theorien entwickeln, sind politisch wie persönlich nicht unbedingt liebenswerte Gestalten. Das ist umso schlimmer bei Weber, weil er anders als Heidegger eine 
explizite politische Theorie entwickelt. Weber hätte es deshalb besser wissen müssen.

\section{Hans-Martin Schönherr-Mann}

Heinz A. RICHTER: Griechenland 1950-1974: Zwischen Demokratie und Diktatur, Mainz/Rubpolding: Verlag Franz Philipp Rutzen, in Kommission bei: Harrassowitz Verlag, Wiesbaden, 2013, Reibe: Peleus - Studien zur Archäologie und Geschichte Griechenlands und Zyperns, Band 60, 501 Seiten, gebunden, $49 €$

Nach dem Standardwerk Die Zeit der Bürgerkriege (2012) widmet sich der emeritierte Professor für griechische und zypriotische Zeitgeschichte an der Universität Mannheim Heinz A. Richter nun dem Griechenland des darauf folgenden Vierteljahrhunderts. Ohne Einschränkung darf auch Zwischen Demokratie und Diktatur als erste wissenschaftlich fundierte und umfassende Darstellung dieser Epoche in deutscher Sprache gelten. Das Buch setzt ein mit den ersten Nach-Bürgerkriegswahlen im März 1950 und einer für Griechenland bis heute tragischen Erkenntnis: "Während alle anderen Staaten Europas, die in den Zweiten Weltkrieg verwickelt gewesen waren, 1945 den Wiederaufbau (...) begannen, erlebte Griechenland zwischen 1946 und 1949 ein noch schlimmeres Desaster als den Zweiten Weltkrieg, einen Bürgerkrieg, der das Land völlig ruinierte. « Zudem sorgten die Interventionen der Briten und US-Amerikaner dafür, dass »das Krebsübel der griechischen Gesellschaft, der Klientelismus, « auch nach den Kriegen wieder restauriert wurde. Die Ausgangsbedingungen für eine demokratische Nachkriegsentwicklung mit politischen Strukturen, die denen in westeuropäischen Staaten entsprochen hätten, waren also denkbar schlecht.

Dennoch siegten bei den Wahlen von 1950 die liberalen Kräfte. Richter führt dies darauf zurück, dass die Bevölkerung eine Aussöhnung und Demokratisierung der Gesellschaft wollte. Beides scheiterte an internen und externen Faktoren: Washington fuhr die finanzielle Hilfe für Griechenland durch den Marshall-Plan exakt zu einem Zeitpunkt herunter als das Land sie am nötigsten gebraucht hätte. Zudem konnte Griechenland nach Bürgerkriegsende seine militärischen Ausgaben durch den Beginn des Koreakriegs kaum vermindern. Aus Furcht vor der kommunistischen Ge- fahr »musste Griechenland überproportionale Streitkräfte unterhalten. Die Folge war Schuldenmacherei.« Der Koreakrieg führte letztlich auch dazu, dass die USA nicht mehr die Liberalen, sondern die griechische Rechte unterstützte. Man förderte den Aufbau einer rechten Sammlungsbewegung, den Synagermós, und sorgte mit Hilfe der Einführung des Mehrheitswahlrechts für dessen Wahlsieg im November 1952. Richter urteilt pointiert: »Nach der Machtübernahme durch Papágos 1952 verschwand das bisschen Demokratie, das Plastíras eingeführt hatte. Der Staat wurde von allen liberalen Elementen gesäubert und der Überwachungsapparat ausgebaut.«

Nach Papágos Tod 1955 wurde Karamanlís Ministerpräsident und stand vor zwei großen Problemen: Er musste das Land wirtschaftlich voranbringen und die wachsenden Animositäten mit den Türken wegen des Zypernproblems in den Griff bekommen. Für den Wiederaufbau brauchte das Land vor allem finanzielle Hilfe, aber von den Amerikanern war nichts mehr zu erwarten. Nachdem aber 1957 die Bundesrepublik Deutschland 300 Mio. DM als getarnte Reparationen an Jugoslawien zahlte, sah Griechenland seine Chance, ebenfalls an deutsches Geld zu kommen. Als Bonn versuchte, die griechischen Forderungen zu verschleppen, kidnappte Griechenland den ehemaligen deutschen Kriegsverwaltungsrat Max Merten als Faustpfand und stilisierte ihn zu einem Kriegsverbrecher, der er in Wahrheit nie war. Die Bundesrepublik gab der griechischen Erpressung nach und zahlte Ende 1958 schließlich 315 Mio. DM an Griechenland, getarnt als Wiederaufbaukredite und Entschädigungen an die griechischen Juden. »Aber nur ein geringer Teil der Summe erreichte die Opfer«, bilanziert Richter. Selbstverständlich reichten aber auch die deutschen Zahlungen nicht aus, um aus dem agrarisch geprägten Entwicklungsland ein industrielles Schwellenland zu machen. Jahre zuvor war dies auch der Grund, weshalb die USA ihre Finanzhilfe einstellten: Man hielt Griechenland für einen »aussichtslosen Fall«. Eine Einschätzung, die heute - mehr als 50 Jahre später - wieder an bedrückender Aktualität gewinnt.

Richter zeichnet die politischen Entscheidungszusammenhänge in anschaulicher und spannender Weise akribisch und kenntnisreich nach, kaum ein Detail bleibt unerwähnt. Manchmal erinnert der Stil an eine Chronologie. Auch die Be- 
ziehungen zur Bundesrepublik Deutschland werden immer wieder angesprochen. Die Gesamtbetrachtung am Ende wirft kein gutes Licht auf das Land und seine politische Führung: »Von außen betrachtet erschien Griechenland als eine parlamentarische Monarchie, war aber weit davon entfernt, eine Demokratie westeuropäischer Prägung zu sein. Die Parlamentarier vertraten nicht den Volkswillen, sondern den ihres Parteichefs. (...) Das Resultat war eine enge Verflechtung der politischen und ökonomischen Oligarchie. (...) Mit Demokratie hatte all das wenig gemein.« Der Klientelismus wurde auch in der kurzen Regierungszeit von Geórgios Papandréou von November 1963 bis Juli 1965 nicht angetastet. Im Gegenteil, er verstärkte zudem die Putschneigungen der Armee umso mehr.

Im Gegensatz zum wissenschaftlichen Mainstream hält Richter den Putsch der Obristen um Papadópoulos im April 1967 für eine »rein griechische Angelegenheit « ohne Kenntnis oder gar Beteiligung der USA. Auch die Junta trat an, das klientelistische System Griechenlands zu überwinden und auch sie scheiterte daran. Im Gegenteil, die Militärs und ihr Umfeld entwickelten sich zu einer neuen Klientel. Den Putsch selbst schätzt Richter nicht als nationale Katastrophe ein, denn es gab in diesen Jahren einen wirtschaftlichen Aufschwung und der Tourismus kam langsam auf Touren. »Wer unpolitisch war, konnte damals ganz gut leben «, bilanziert Richter. Die Katastrophe begann mit dem Studentenaufstand im November 1973, in dessen Folge die Junta außenpolitische Erfolge zur Legitimierung ihrer Macht suchte und in völliger Verkennung der Lage die Zypernkrise auslöste. Das Ergebnis war die türkische Invasion und die Teilung der Insel, die bis heute andauert.

Richters Fazit nach dem Untergang der Obristenherrschaft und der Wiederherstellung des Ancien Regimes unter Karamanlís fällt ernüchternd aus: Während in Spanien und Portugal »etwa zur gleichen Zeit Diktaturen beendet wurden und demokratische Strukturen entstanden, blieb in Griechenland hinter der demokratischen Fassade alles beim Alten. «Die Abschaffung der Monarchie mittels eines Plebiszites 1974 hält Richter für reine Kosmetik. »Das klientelistische System lebte munter weiter und verfestigte sich immer mehr. «Die Schulden des Staates stiegen kontinuierlich weiter an, doch die Bevölkerung hatte den Eindruck, dass es aufwärts ging und war zufrieden. In keiner der historischen Umbruchphasen - weder jene nach dem Bürgerkrieg, noch jene während oder nach der Junta - gelang es, den Klientelismus zu brechen. In der Folge übertraf der Zugriff auf die staatlichen Mittel alles Vorangegangene und setzte neue Maßstäbe bei Betrug und Korruption. »Der Endpunkt dieser Entwicklung ist bekannt: die Krise im zweiten Jahrzehnt des 21. Jahrhunderts.« Dem kann nicht widersprochen werden.

\section{Dirk Schönrock}

Paul SÖRENSEN und Nikolai MÜNCH (Hrsg.): Politische Theorie und das Denken Heideggers, Bielefeld: transcript Verlag, 2013, 252 S., 29,80 €

Die zentrale »Frage « des Buchs lautet, »ob und (gegebenenfalls) inwiefern in systematischer Hinsicht von Seiten der politischen Theorie an das heideggersche Denken angeknüpft werden kann.« (12) Die Beantwortung dieser Frage erfolgt aus zwei Perspektiven: Im ersten Teil des Buchs, der den Titel »Motive« trägt, soll den »zentralen Fragen und Motiven der politischen Philosophie und Theorie in Heideggers Denken « nachgespürt werden; in den zweiten Teil, »Perspektiven « genannt, sind »Untersuchungen aufgenommen, die sich im weitesten Sinne einer Politischen Philosophie und Theorie nach Heidegger widmen «. (13) Die von den beiden Herausgebern zusammengetragenen Aufsätze sollen also zum einen klären, inwiefern sich aus dem verschriftlichten Denken Heideggers heraus Anschlussmöglichkeiten für eine Politische Philosophie zeigen bzw. überhaupt zeigen können und zum anderen inwiefern sie als Impulse bereits wirkten und (weiter-)wirken. Diese strikte Trennung von Möglichkeit und Faktizität ist im Buch jedoch nicht stringent durchgehalten und wohl auch nicht durchzuhalten. Denn Motive sollen entdeckt und Perspektiven aufgezeigt werden: Motive und Perspektiven stehen folglich in einem hermeneutischen, intentionalen Zusammenhang. Schade ist, dass das Buch keinen dritten Teil hat, keine Synthese des Zusammenspiels der herausgearbeiteten Motive und Perspektiven versucht. Die Herausgeber beschränkten sich lediglich darauf, Aufsätze zu sammeln und unter einen der beiden Buchteile zu subsummieren.

Zunächst ist festzuhalten, dass eine mögliche Anknüpfung an Heidegger - insbesondere unter 
philosophisch-politischer Rücksicht - bereits »angesichts des unsäglichen politischen Engagements [Heideggers] im nationalsozialistischen Deutschland « desavouiert scheint. (7)

Gleichwohl zeigen sich durch alle Schaffensperioden hindurch politisch (bislang) unverdächtige Anknüpfungspunkte. Diese kommen bei Heidegger jedoch nicht zum Tragen; sie nehmen als Motive im Kontext der jeweiligen Arbeit, bzw. des Gesamtœvres in politischer Perspektive einen nur marginalen Platz ein. Leider gehen die Aufsätze kaum darauf ein, warum Heideggers Texte so auffällig apolitisch verfasst sind/zu sein scheinen.

Im ersten Beitrag versucht Grosser »Heideggers Ausführungen zu »Mitsein" und »Mitdasein « für relevante politik- und sozialtheoretische Diskurse der Gegenwart fruchtbar zu machen«. (21) Er konstatiert im Schnelldurchgang durch alle Schaffensperioden hindurch »ebenso ausbaufähige wie ausbaubedürftige Anlagen« eines »plurale[n] Gemeinschafts-Modell[s] «. (39) Worin dieses bestehen könnte bleibt jedoch vage. Insbesondere die für Heidegger so bedeutsamen Bezüge auf Zeitlichkeit und Sprache sind nicht mitreflektiert. Der zweite Beitrag von Siegfried befasst sich mit der »Freiheitsthematik bei Heidegger «. (43). Die Autorin differenziert den Freiheitsbegriff in »drei Rezeptionslinien « aus und möchte aufzeigen, dass »in aktuellen Diskussionen " sowohl die "politische als auch die "ethische « und »ontologische« Linie »präsent sind «. (45) Warum ihre Arbeit dem ersten Teil des Buchs zugeordnet ist, ist insofern fraglich, als sie von der Perspektive, der Rezeption ausgeht. Im dritten Beitrag behauptet Tömmel, dass der »liebende Streit $[\ldots]$ die einzige vielversprechende Konzeption von Mitsein bei Heidegger « sei. (76) Gleichwohl ist ihrer Ansicht nach aufgrund des »Mangel[s] an Kommunikation [... in] Heideggers Philosophie « dessen »Position [...] für die Gegenwart letztlich unattraktiv «. (77) Im vierten Beitrag stellt Reitz die »Frage, inwiefern Ästhetik politisch Geschichte machen kann.« (82) Auch er konstatiert, dass eine "grundsätzliche Korrektur an Heidegger « nötig sei (100) und erachtet (statt Heidegger) die Konzeption des späten Benjamin als » pionierhaft «. (101) Im letzten Aufsatz des ersten Teils soll »Heideggers Auffassung von der Menschenwürde im Hinblick auf die von ihm vorgetragene Metaphysikkritik « herausgearbeitet werden. (106) Bruns sieht den »Grund dafür, dass die Würde des Menschen unantastbar ist, [...in] dessen Existenz«. (127) Heideggers Existenzialphilosophie erscheint so zumindest für die Begründung der Menschenwürde als anschlussfähig.

Im ersten Aufsatz des zweiten Teils geht Meinefeld auf Arendts Man-Analyse in Sein und Zeit ein, um »im Rekurs auf »Jedermann « [...] das öffentliche Miteinandersein als eine eigentümliche Möglichkeit menschlichen Lebens verständlich zu machen. (140) Es komme Arendt dabei darauf an, »das »eigentliche Miteinander « [so] zu erschließen [...], dass die unterschiedlichen Perspektiven [in Divergenz zu Heidegger] als eine Pluralität zur Geltung kommen.«(147) Im zweiten Aufsatz untersucht Wesche »Heideggers Einwände gegen die Demokratie«. (153). Wesches Ansicht nach »fallen [in der Demokratietheorie] drei Einsichten Heideggers auf fruchtbaren Boden. Erstens müssen Demokratien der Diagnose von Selbsttäuschungen [...] Rechnung tragen.« (167) Zweitens »kann [...] Heideggers Gedanke der Sprachpraktiken fruchtbar gemacht werden «, indem »die sprachlich verfasste Lebenswelt von Bürgerinnen und Bürgern an die Stelle der Geistesgemeinschaft von Dichtern und Denkern « trete. (169) Drittens zeige »Heideggers Einsicht in die Kontingenz der rationalisierenden $\mathrm{Kraft}$ « das Problematische in der »Verschiebung von der Geistesgemeinschaft zur Zivilgesellschaft«. (169) Wie von Heidegger existenzialontologisch nüchtern formuliert, müsse man es dem Bürger überlassen, »ob er sich mit trivialen, voreingenommenen und verzerrten Meinungen begnügt oder ob er reflektierten Meinungen einen Vorrang einräumt. « (170) Im Anschluss an Wesche beschäftigt sich Flügel-Martinsen in »Auseinandersetzung mit Marchart« (178) mit »Linksheideggerianismus als prima philosophia «. (178) Den Ausgangspunkt hierfür bilde in Marcharts postfundamentalistischem Ansatz die »zentrale Differenz zwischen Politik und Politischem [...] in Analogie zur ontologischen Differenz«. (179) Problematisch werde diese "Erzählung«, (179) wenn sie, statt die »Versuche einer Festlegung der sozialen und politischen Welt ad absurdum zu führen, [...] eine Ontologie des Politischen [entwirft], die meint, genau angeben zu können, worin das Politische besteht.«(188) Im vorletzten Aufsatz des zweiten Teils thematisiert Taylor den Bezug von »Sprache und Ökologie«. (191) Für ihn ist Heidegger "ein Konstitutionstheoretiker". (206) Denn die »Sprache erschließt«, insofern »sie 
hilft, den von Menschen geteilten Raum zu bestimmen.« (206) Dieses Verständnis von Sprache als Lichtung bilde daher »die Basis einer Tiefenökologie«: (222) »Unsere Ziele sind bestimmt durch etwas, demgegenüber wir uns eigentlich als dienend verstehen sollten. (223) Im letzten Buchbeitrag beschäftigt sich Hollendung mit Butlers Politik der Verletzbarkeit. Im Zuge ihrer Analyse stellt die Autorin fest, dass sich »in Butlers Beschreibung der Sozialität des Subjekts das >Mitsein Heideggers widerspiegelt.« (237) Doch Heideggers »Vernachlässigung des Alltäglichen und sein Desinteresse an Fragen des Politischen ließen ihn die wesentlichen Konsequenzen, die sich aus seinen Überlegungen zum >Mitsein< ergeben, übersehen.« (243)

Fazit: Das Buch bietet eine Vielzahl an Ansatzpunkten (auf die hier nur sehr beschränkt eingegangen werden kann), um eine weiterführende Diskussion anzuregen. Allerdings zeigt sich sehr deutlich, dass die Anschlussmöglichkeiten (insbesondere am Existenzial des Mitseins oder der Sprache als Lichtung des Seins) dadurch restringiert sind, dass Heidegger selbst das Politische seiner Philosophie in seinen Veröffentlichungen (wohl bewusst und kalkuliert) ausblendete.

Auf Heideggers »seinsgeschichtlichen Antisemitismus « (Trawny) wird nicht eingegangen. Die Veröffentlichung der Schwarzen Hefte im Zuge der Gesamtausgabe dürfte hierzu jedoch noch reichlich Zündstoff liefern und die Auseinandersetzung mit Heidegger, gerade in politischer Hinsicht, neu entfachen.

In allen Aufsätzen völlig ausgeklammert ist zudem der Bezug von Mitsein zu Jemeinigkeit. Hierdurch wird der Mitsein-Gedanke zudem überzeichnet und überbewertet.

Lohnend aber ist die Beschäftigung mit Heidegger, so zeigt das Buch, ohnehin nicht so sehr, um eine Theorie des Politischen zu fundieren, was aus Heideggers Werk heraus wie gezeigt kaum möglich ist. Vielmehr hilft die Auseinandersetzung mit Heidegger, um eine ausformulierte Theorie des Politischen entsprechend abzusichern, bzw. darauf hin zu untersuchen, ob und inwiefern sie die durch Heideggers Philosophie eröffneten Perspektiven existenzial- und sprachontologischer Fragestellungen angemessen mit zur Sprache bringt.

Georg Simet
Bernhard SUTOR: Katholische Soziallehre als politische Ethik. Leistungen und Defizite, Paderborn u. a.: Ferdinand Schöningh, 2013, 296 S., 39,90 $€$

Dem politisch bzw. politikwissenschaftlich Interessierten sind sehr wahrscheinlich das Subsidiaritätsprinzip und das Solidaritätsprinzip geläufig. Dass es sich dabei um grundlegende Prinzipien der Katholischen Soziallehre handelt, dürfte dagegen wohl nicht jedermann bekannt sein. Gleichwohl bilden beide Vorstellungen geradezu allgemeingültige Handlungsmaximen im Bereich der Politik. Kann die Katholische Soziallehre daher die Grundlage für eine politische Ethik bilden? Mit dieser Frage beschäftigt sich Bernhard Sutor seit Jahrzehnten. Der vorliegende Band umfasst Aufsätze aus gut dreißig Jahren, die diese Problematik anhand grundsätzlicher Überlegungen, aber vor allem aus der Perspektive konkreter Fragestellungen thematisieren. Es geht dabei um das Verhältnis zum liberal-demokratischen Rechtsstaat, um das Problemfeld der sozialen und politischen Gerechtigkeit und um die Frage nach der Ethik in den internationalen Beziehungen.

Der Autor setzt die Soziallehre nicht mit einer politischen Ethik in eins, sondern richtet den Blick zwar wohlwollend aber durchaus kritisch auf die Christliche Soziallehre und deren Nutzen für die Politik. Daher inspiziert er zunächst das Spannungsfeld, das zwischen der Sphäre des Politischen und der kirchlichen Lehre besteht. Vor allem zwei Aspekte scheinen hier auf. Die Katholische Soziallehre neigt - wie jede Ethik - dazu, politisches Handeln zu stark moralisch aufladen zu wollen. Damit läuft man allerdings Gefahr, die Politik zu überlasten. Die politische Praxis ist ausgerichtet auf die Auseinandersetzung um die richtige Lösung und den Kompromiss, der Maximalforderungen abschleift und damit aus der Sicht einer rein moralisch-ethischen Betrachtungsweise immer defizitär erscheinen muss. Auch lassen sich politische Forderungen und Vorstellungen, die sich dem Kompromiss als Handlungs- und Entscheidungsoption systematisch verschließen, leicht als Basis für fundamentalistische und damit intolerante Positionen missbrauchen. Dennoch ist es für den $\mathrm{Au}$ tor unstrittig, dass politisches Handeln einen ethischen Maßstab benötigt. Es ist die Balance zwischen Machbarkeit auf der einen und Verantwortlichkeit auf der anderen Seite, die gewahrt werden sollte. Ganz nebenbei eignet sich dieser Stand- 
punkt als pragmatische Lösung des immer noch ausgetragenen Konflikts zwischen Macht und Ethik in der politischen Theorie. Man kann Bernhard Sutor nicht im Sinne von Raymond Geuss vorwerfen, dass er die Macht als zentrales politisches Motiv negiert und sich einer mehr oder minder naiven »Ethik-hat-Vorrang-Regel « bedient. Er stellt sich der Machtfrage, wobei er seinerseits nicht den Fehler macht, damit die ethische Dimension des Politischen kleinzureden oder sogar ganz auszublenden. Als zweiten Aspekt merkt der Autor an, dass die klassische kirchliche Gesellschaftslehre nicht sonderlich aussagekräftig ist, wenn es um die spezifische Funktionsweise von politischen Institutionen in demokratischen Systemen geht. Diese Frage wird in den Sozialenzyliken nicht ihrer Bedeutung gemäß behandelt. Moralische Appelle an den Einzelnen sind aber zu wenig, um die Stellung von Institutionen bei der Formulierung und Umsetzung politischer Ziele angemessen zu würdigen. Dieser Befund mag verwundern, wurde und wird doch innerkirchlich über »institutionelle Gewalt « oder die »Strukturen der Sünde" debattiert; ein positives Verhältnis zu den staatlich-demokratischen Institutionen ist damit aber nicht automatisch verbunden. Wie steht es nun mit dem katholischen Anspruch, Politik zwar nicht machen, aber doch ermöglichen zu wollen? Auch das ist im Übrigen kein geringes Anliegen. Es kann umgesetzt werden, aber nur unter der Bedingung, dass der Kern der Politischen nicht verkannt und verfehlt wird. Bernhard Sutor bringt deshalb durchaus auf der Basis der theologischen Tradition - die Klugheit ins Spiel. Damit widmet er sich einer Kategorie, die leider etwas von der Bildfläche der politischen Theorie verschwunden zu sein scheint. Der Nutzen der Klugheit für eine ethisch grundierte Politik liegt an erster Stelle in der Möglichkeit, pragmatisches Handeln einzufordern. Nicht durch die starre, kompromisslose Durchsetzung ethischer Maximen wird eine am Gemeinwohl orientierte Ordnung geschaffen, sondern durch situationsbezogene und konkrete Lösungsvorschläge.

Die katholische Kirche ist historisch gesehen kein natürlicher Verbündeter der liberalen Demokratie. Vielmehr stand lange Zeit eine antimodernistische Abwehrhaltung gegen die Errungenschaften der Demokratisierungsbewegungen im 19. und 20. Jahrhundert im Vordergrund. Es bedurfte großer Anstrengungen und Bemühungen, um die freilich auch systematisch angelegten Unterschiede zwischen kirchlicher Lehre und liberalem Menschenbild und Gesellschaftsverständnis in konstruktive Modelle des Dialogs und des Miteinanders umzuformen. Für die Gegenwart heißt das: »Die Christliche Soziallehre katholischer Prägung tritt heute rückhaltlos für Demokratie ein, besteht aber darauf, dass sie einer philosophischen Begründung über die bloße Rechtssetzung hinaus bedarf« (93). Dies lässt sich anhand zweier Gesichtspunkte illustrieren. Zum einen gilt, dass pluralistisch organisierte Gemeinwesen keine relativistischen Systeme darstellen. Plurale Formen des Konfliktaustrags sind angewiesen auf die Anerkenntnis des Anderen als gleichberechtigten Mitspieler, die sich auf ihn selbst und seine Meinungen und Standpunkte erstreckt. Sie ist die Basis für Toleranz, ohne die eine politische Gemeinschaft keinen Diskurs über das Gemeinwohl führen kann. Auch an dieser Stelle verweist der Autor auf die entsprechenden theologischen Traditionslinien. Das jüdisch-christlich geprägte Menschenbild scheint einen gut passenden Rahmen für die ethischen und philosophischen Anforderungen zu liefern, die mit der Begründung einer modernen Demokratie verbunden sind. Ein zweiter Punkt greift die Ambivalenz der menschlichen Natur auf. In der jüdischen sowie christlichen Theologie ist der Mensch nicht nur mit seinen positiven Eigenschaften präsent. Seine moralische Inkonsistenz und sein oftmaliges Scheitern in vielen Belangen seines privaten, beruflichen und öffentlichen Lebens werden nicht ausgespart. Ganz im Gegenteil bildet die Unzulänglichkeit von uns Menschen einen konstituierenden Faktor für die gesamte Lehre. Der fehlbare Mensch ist nicht in der Lage, unfehlbare Ordnungen zu schaffen und zu erhalten. Menschliches Maß ist von Vorläufigkeit und Endlichkeit geprägt. Für die Politik bedeutet dies, dass keine idealen Zustände erreicht werden können und Vorstellungen keine letzte Gültigkeit für sich beanspruchen dürfen. Politischen Maßnahmen müssen revidierbar sein. Es existiert in dieser Sphäre weder etwas Alternativloses noch Unumkehrbares. Nur demokratisch-plural verfasste Gemeinwesen können die angesprochene Zwiespältigkeit des menschlichen Wesens adäquat institutionell und normativ abbilden.

Gerechtigkeit zählt zu den politischen und theologisch-philosophischen Schlüsselbegriffen. Darum zählt diese klassische Tugend auch zu den 
wichtigsten Gegenständen des vorliegenden Bandes. Schon in den grundsätzlichen Überlegungen $\mathrm{zu}$ Beginn weist Bernhard Sutor darauf hin, dass ohne die Vorstellung von Gerechtigkeit und einer gerechten Ordnung keine Gemeinwohlkonzeption entworfen werden kann. Doch Gerechtigkeit ist ein hoch umstrittener Begriff. Jede politische Ideologie, jede Richtung und jede Partei entwickelte eigene, oftmals sehr verschiedene und sich widersprechende Definitionen und Bestimmungen davon, was gerecht sein könnte. In der derzeitigen Debatte steht die »soziale Gerechtigkeit « häufig im Vordergrund. Die Katholische Soziallehre darf sich dem politischen Streit nicht entziehen, sonst bleibt ihr nur der moralische Zeigefinger als auf die Dauer wirkungslose Geste. Die Frage nach der sozialen Gerechtigkeit ist deshalb die Frage nach den Strukturen und Institutionen, in deren Rahmen sie verwirklicht werden kann: »Wir vertreten hier die These, dass die Redeweise von sozialer Gerechtigkeit theoretisch-systematisch nur dann einen Sinn hat, wenn man in ihr nicht eine neue Variante oder eine Kombination von Dimensionen der alten Tugendlehre sieht; wenn man sie vielmehr als Ausdruck der Frage nach den richtigen sozialen Institutionen versteht « (159). Damit ist jedoch das Problem der Gerechtigkeit nicht abschließend geklärt. Es bedarf der Ergänzung der sozialen Gerechtigkeit um die politische Gerechtigkeit, die der Autor im Anschluss an Otfried Höffe als eine Ordnung begreift, in der »eine für alle Beteiligten nach Gerechtigkeitsregeln annehmbare politische Ordnung " (164) herrscht. Beide Ausprägungen der Gerechtigkeit müssen sich gegenseitig ergänzend umgesetzt werden. Dies bedarf der Teilhabe am wirtschaftlichen, kulturellen und politischen Leben der Gesellschaft. Doch gerade hier zeigt sich nochmals das Defizit der kirchlichen Soziallehre, das in der Vernachlässigung der Institutionen besteht. Bernhard Sutor plädiert dafür, dass die Soziallehre stärker als bisher die strukturellen Erfordernisse der eigenen Grundsätze und Postulate in den Fokus rückt. Problematisch wird die moralische Bewertung der Gerechtigkeitsfrage, wenn darüber der Aspekt der Leistungsfähigkeit des Staates und der Wirtschaft aus den Augen verloren wird. Der Autor nimmt die Katholische Soziallehre stärker beim Wort, als dies z B. im gemeinsamen Papier der beiden großen Kirchen in Deutschland zur wirtschaftlichen und sozialen Lage aus dem Jahre 1994 der Fall ist, wenn er die Bedeutung des Subsidiaritätsprinzips unterstreicht, das die Eigenverantwortlichkeit des Einzelnen in Rechnung stellt, bevor das komplementäre Solidaritätsprinzip und damit die Gemeinschaft aller Bürgerinnen und Bürger in Anspruch genommen werden kann. Bernhard Sutor schlägt eine stärkere Berücksichtigung der Beteiligungsgerechtigkeit vor. Damit ist ein Konzept verbunden, das nicht nur den barrierefreien Zugang zur Partizipation ermöglicht, sondern zugleich die Freiheit gewährleistet, die sich dadurch eröffnenden Chancen ohne Zwang zu ergreifen. Gerechtigkeit ist nicht nur in der autoritativen Verteilung von Gütern zu finden. Ebenso wichtig erscheint die institutionelle Bereitstellung von Teilhabeoptionen, insbesondere dann, wenn der Sozialstaat an seine finanziellen Grenzen stößt. Alle Reformbemühungen im sozialstaatlichen Bereich sollten den »Vorrang « (207) der Beteiligung vor der Umverteilung widerspiegeln.

Die internationalen Beziehungen bilden den Schlusspunkt der Betrachtungen. Die Dynamik der Globalisierung und deren nicht zu unterschätzenden Auswirkungen auf innerstaatliche politische Entscheidungen und Prozesse machen es notwendig, darauf aus der Perspektive der Katholischen Soziallehre Antworten zu finden. Um diese $\mathrm{zu}$ formulieren und in den gesamtgesellschaftlichen Diskurs einzubringen, sollte die Kirche mit all ihren vielfältigen Organisationen vernetzt tätig werden, um die Christliche Gesellschaftslehre mit dem politischen Handeln der Christen wieder neu zu verbinden. Auch die Verknüpfung mit den profanen Wissenschaften könnte dazu führen, dass die Soziallehre wieder verstärkt in der öffentlichen Debatte wahrgenommen wird. In der Phase des Kalten Krieges war die Ausarbeitung einer umfassenden Friedensethik ein wichtiges Anliegen des kirchlichen Lehramtes. Es ging allerdings nicht um eine einfache Entgegensetzung von positivem und negativem Frieden, da eine ewige Friedensordnung kein von Menschen erreichbares Ziel darstellt, wohl aber um ein Werben für internationale Ordnungsstrukturen, die eine friedliche Konfliktlösung zu befördern vermögen. Damit gehen nach Bernhard Sutor drei fundamentale Vorstellungen einher: Die Förderung der Menschenrechte, das Eintreten für den liberalen Rechtsstaat und die Pflicht, unsere materiellen Bedürfnisse bezogen auf Umweltverträglichkeit und Solidarität zu verringern. In der Zeit der nuklearen Abschreckung 
empfahl der Autor die Strategie des Gradualismus, der eine Abfolge von vertrauensbildenden Maßnahmen vorsah. Dieses System von Vorleistungen und Gegenleistungen hat angesichts der heutigen Krisen wenig an Aktualität verloren. Wieder geht es nicht um eine Missachtung der politischen Machtpotentiale, sehr wohl aber um deren institutionelle Einschränkung und Kontrolle. »Friedenssicherung besteht nicht im Abbau von Macht, sondern im Versuch, durch kluge Disposition von Macht Gewalt auszuschalten " (275). Im letzten Abschnitt des Buches wendet sich Bernhard Sutor den humanitären militärischen Interventionen zu, deren Legitimität er anhand der klassischen Kriterien der Lehre vom gerechten Krieg durchspielt. Zwar lassen sich heute noch humanitäre Interventionen damit begründen, jedoch muss die jeweilige Situation genau analysiert werden, um eine Umsetzung der Kriterien im Sinne einer wirklich humanitären Aktion zu bewerkstelligen. Bernhard Sutor listet am Beispiel der Erfolgsaussicht militärischer Einsätze eine Reihe von Bedingungen auf, die von der intervenierenden Partei erfüllt werden müssen. Das sind beispielsweise neben der Berücksichtigung kultureller Eigenheiten, die Bereitstellung von Wiederaufbauhilfen und die Entwicklung konflikthemmender politischer Strukturen. Es genügt somit nicht, einfach einen Konflikt gewaltsam zu beenden, es muss zusätzlich die Bereitschaft vorhanden zu sein, sich darüber hinaus langfristig zu engagieren, um eine tatsächliche Befriedung zu erreichen. Die Entwicklungen in Afghanistan zeigen deutlich, dass dies keine einfache Unternehmung ist. Dessen ungeachtet hat die internationale Gemeinschaft die Pflicht, bei schweren Menschenrechtsverletzungen einzugreifen und im Notfall dafür auch militärische Mittel einzusetzen. Letztlich ist aber der Aufbau eines »Friedensföderalismus der Staaten « (293) unabdingbar. Hierin ist der kirchlichen Lehre zu folgen.

Trotz der vom Autor formulierten Kritik an der Katholischen Soziallehre, zeigt Bernhard Sutor eindrucksvoll, warum politisches Handeln eine Praxis darstellt, die ohne ein ethisches Fundament nicht auskommen kann. Die Bezugnahme auf die Katholische Soziallehre stellt diesbezüglich eine ethisch gut zu begründende und sinnvolle Wahl dar. Die vorliegende Aufsatzsammlung kann als sehr stimmige Ein- und Weiterführung in die behandelte Thematik verwendet werden. Es ist zu wünschen, dass sie nicht nur ihren Weg in die uni- versitären Bibliotheken sondern auch in die Redaktionsstuben und nicht zuletzt auf die Schreibtische kirchlicher Amtsträger findet.

Bernhard Schreyer

Edgar WOLFRUM: Rot-Grün an der Macht. Deutschland 1998-2005, München: C. H. Beck, 2013, 848 S., 24,95 $€$

Die bundesrepublikanische Nachkriegsgeschichte wird als intrikate Topographie von langen Linien der Kontinuität und einer Vielzahl kleinerer Brüche deutlich. Gelernt hat das Land, seine eigenen Maßstäbe zu entwerfen, sie im öffentlichen Streit $\mathrm{zu}$ korrigieren und weiterzuentwickeln. Jede Bundesregierung hat bislang daran ihren Anteil gehabt und steht inmitten dieser Verlaufsgeschichte. Mitunter vollzieht die Politik Entwicklungen, die in der Gesellschaft schon längst angekommen sind.

Ein wenig war dies der Fall, als am 27. September 1998 die Kanzlerschaft Helmut Kohls zu Ende ging und eine rot-grüne Bundesregierung ins Amt $\mathrm{kam}$. SPD und Grüne hatten viele Ideen, die sie ins Bonner Kanzleramt mitbrachten. Sie wussten, dass sie es endlich geschafft und nun an der Macht die Gelegenheit hatten, ihre Politik gemeinsam umzusetzen. Über den großen Plan, das grand design verfügte Rot-Grün damals nicht, wohl aber war die Regierung getragen von der Vorstellung, weitreichende gesellschaftliche Veränderungen ins Werk zu setzen: ökologisch nachhaltig, friedlich, modern, liberal und wegweisend ins 21. Jahrhundert - all das waren die Attribute, die fortan die Politik bestimmten. Aus der Rückschau betrachtet, liegt hier eines der Missverständnisse der rotgrünen Regierungszeit begründet: Den Kurs, den Rot-Grün der Gesellschaft verordnete, hatte das Land im Grunde schon längst eingeschlagen: Die Republik hatte sich über Jahrzehnte hinweg als wandlungs- und anpassungsfähig erwiesen. In ständigem Selbstgespräch begriffen, hatte das Land gelernt, Konflikte auszutragen und im diskursiven Streit nach Lösungen zu suchen - die politische Kontroverse als Treibsatz für gesellschaftliche Veränderungen hatte die Republik verinnerlicht. Insofern war das Visionäre, das im Herbst 1998 in der Luft lag, in Wahrheit ein gutes Stück Bestätigung vom Wesen der Bonner Republik.

Edgar Wolfrum nimmt mit seinem 800 Seiten umfassenden Werk eine Verortung der rot-grünen 
Bundesregierung in den weiten Entwicklungsbewegungen des vereinten Deutschlands vor. Plastisch wird das Spannungsverhältnis von innenpolitischer Richtungsgebung und multilateraler Einbindung auf dem Feld der Außenpolitik. Wolfrum gelingt es meisterhaft, die jüngste Zeitgeschichte zum Schwingen zu bringen. Die rot-grünen Regierungsjahre werden in ihrem gesamten Facettenreichtum und ihrer bisweilen dramatischen Ereignisdichte noch einmal erlebbar.

Rot-Grün hatte von Anfang an schweres Gepäck bei sich: Von vielen Beobachtern zum »Projekt « stilisiert, ging die neue Koalition gleichsam mit einem normativen Überschuss ans Werk. »Neue Mitte sowie »Innovation und soziale Gerechtigkeit « dienten als Zauberformeln, um die Wähler zu überzeugen. Kaum im Amt angekommen, holten Bundeskanzler Gerhard Schröder und Außenminister Joschka Fischer zur großen Geste aus; alles war auf Neuanfang programmiert, alles sollte angepackt werden, und zwar sofort und immer mit dem Anspruch versehen, es besser zu machen als die Vorgängerregierung. Jahre ihres politischen Wirkens hatten die Akteure auf diesen Augenblick hingearbeitet. Jetzt wollten sie reüssieren und spürten gleichzeitig die ungeheure Last der Erwartungen.

Die neue Koalition sollte »ein Bündnis für die Innenpolitik sein « (20). Gewiss, vieles war in den letzten Jahren der schwarz-gelben Bundesregierung unter Kanzler Kohl liegengeblieben, das Land hatte an Dynamik verloren. "Dass die Bundesrepublik strukturell reformunfähig sei - dies wollte Rot-Grün widerlegen « (21). Doch das war eine Fehlwahrnehmung der Bundesrepublik: Ein lernendes System, programmiert auf eine ewige Abfolge kleiner Veränderungen, bereit zur Selbstkorrektur. Und gerade hierin liegt eines der vielen Missverständnisse über die Leistungsfähigkeit des politisch-administrativen Systems. Die Diagnose "Reformstau « (21), die sich auch bei Wolfrum findet, taugte Mitte der 90er Jahre allenfalls als populäre Zuspitzung des Augenblicks. Tatsächlich aber ist das Land in steter Bewegung begriffen, zumeist geräuschlos, unspektakulär, ohne Fanfarenstoß. Dieser genetische Code der Politik - die Bewegungen in kleinen Schritten im Schatten dauerhafter Ungewissheit - wurde unter Rot-Grün besonders deutlich: Kaum hatte die Regierung etwas Zeit, die eigentlichen Verhältnisse zu überblicken, den Moment zum Luftholen zu nutzen und die politischen Schwebeteilchen zu ordnen, war sie sogleich wieder Getriebene neuer Turbulenzen.

Schon nach wenigen Seiten wird klar, dass Wolfrum ein beeindruckendes Buch komponiert hat. Gestützt auf eine schier unbändige Fülle an Primär- und Sekundärquellen, wird zudem die gesamte Bandbreite an Literatur zu Rot-Grün berücksichtigt. Neben der exklusiven Einsicht in bislang verschlossene Regierungsdokumente hat Wolfrum darüber hinaus knapp 40 Interviews mit den Akteuren von einst geführt. Das Werk besticht durch die dichte Darstellung der Ereignisse und ist durch und durch empiriegesättigt.

Unverkennbar schreibt hier der Historiker, nicht etwa der Politikwissenschaftler. Im Zentrum des Interesses stehen nicht einzelne Politikfelder; vielmehr geht es um das Bleibende, das Prägende, ja - wenn man das Wort wählen möchte - das Erbe von Rot-Grün. Deshalb trägt die Regierungsbilanz im Untertitel die weiträumigere Verortung »Deutschland 1998-2005«. Wolfrum möchte sein Werk nicht als "grimmige Bilanz« (708) verstanden wissen. Viele Bewertungen sind bald nach dem Ende der rot-grünen Bundesregierung entstanden, die sich vor allem um politische Fehlersuche bemüht haben. Wolfrum legt andere Maßstäbe an. Ihm geht es darum, »die dynamischen Linien dieser Jahre " (708) nachzuzeichnen und den gesamten Zeitabschnitt als "Zeit des Umbruchs" (708) zu deuten. Wolfrums konzises Urteil ist zutreffend: "Das Tempo war hoch, die Turbulenzen waren groß, und Deutschland veränderte sich dadurch in Stil und Status gleichermaßen « (708).

Zwei wichtige Brüche, die als deutliche Momente der Diskontinuität hervortreten, beschreiben diesen Stil- und Substanzwandel in der bisherigen bundesrepublikanischen Entwicklungsgeschichte: Noch bevor die neue Regierung offiziell im Amt vereidigt war, musste sie im Herbst 1998 über Krieg und Frieden entscheiden. Am 24. März 1999 begann der Kampfeinsatz unter Beteiligung der Bundeswehr gegen serbische Stellungen im Kosovo. »Mit diesem Tag ging die Nachkriegszeit für Deutschland zu Ende« (65), so Wolfrums Urteil. Indem sich die Bundesrepublik das erste Mal seit 1945 an militärischen Kampfhandlungen - zumal ohne UN-Mandat - beteiligte, geschah außenpolitisch »etwas qualitativ Neues « (71). Die Regierung stand unter einem beispiellosen Rechtfertigungsdruck. Auf dem Sonderparteitag der Grünen am 13. Mai 1999 begründete Außenminister 
Joschka Fischer die militärische Intervention mit dem Verweis auf Auschwitz. Während es zum Selbstverständnis der alten Bundesrepublik gehört hatte, wegen der deutschen Geschichte auf das Mittel des Militärs zu verzichten, wurden die Erfahrungen mit dem Nationalsozialismus nun » moralisch und gesinnungsethisch aufgeladen, sie wurden aktualisiert und neu codiert « (106).

Als Bundeskanzler Gerhard Schröder am 14. März 2003 ans Rednerpult des Deutschen Bundestags trat und die Eckpunkte der Agenda 2010 vorstellte, ahnte wohl niemand, dass dies einen »Paradigmenwechsel in der deutschen Sozialstaatlichkeit « (544) nach sich ziehen würde. Erst wenige Tage zuvor war das Bündnis für Arbeit für gescheitert erklärt worden, die klassischen tripartistischen Verhandlungsarrangements waren nicht mehr stark und innovationsfähig genug. »Neuer Schwung konnte nur durch neue Formen entstehen « (539). Und genau hier gelang der Regierung eine grundlegende Umprogrammierung der bisherigen Sozialstaatlichkeit - ein wahrer Politikwechsel, der bis dahin für beinahe undenkbar gehalten worden war. Dennoch: Auch die Agenda 2010 war eingebettet in die langen Linien gewachsener Politik und man kann sie »jedoch durchaus auch in der Kontinuität zum bundesdeutschen Wohlfahrtsstaat sehen, lässt sie sich doch als ein Versuch verstehen, ihn zu modernisieren, zu entschlacken, aber letztlich zu bewahren, genauer, seine 'Substanz' zu erhalten « (581).

Am Abend des 18. Septembers 2005 rollte RotGrün auf das Gleis der Geschichte. Zwar hatte Schröders SPD eine fulminante Aufholjagd im Wahlkampf hinbekommen, doch die Wahlsiegerin war Angela Merkel. Wolfrum schildert noch einmal eindrucksvoll die Elefantenrunde der Spitzenkandidaten am Wahlabend. Berauscht von dem unerwartet guten Wahlergebnis der SPD inszenierte Gerhard Schröder »einen Putsch gegen die Wirklichkeit« (704). Im Augenblick der Abwahl seiner Regierung zelebrierte er seinen persönli- chen Sieg. Doch in Wahrheit vollzog er »eine rotgrüne Selbstentsorgung « (708). Es war ein Abgang mit Aplomb - ganz nach Schröders Art.

Klar wird bei der Lektüre von Edgar Wolfrums Panorama der Zeitgeschichte, wie sehr die sieben Jahre »eine Zeit gesteigerter Reformtätigkeit « (709) waren. In Gerhard Schröders eigenen Worten waren es Jahre, »die unser Land nach innen liberaler, toleranter, sicherer und demokratischer und nach außen selbstbewusster, freier und geachteter gemacht haben « (690). So resümierte Schröder am 1. Juli 2005 als er im Bundestag die Vertrauensfrage stellte. Dies war gewiss zu einem Zeitpunkt, als sich viele Projekte noch im Fluss befanden und die Geschichte noch nicht genügend Abstand für eine kritische, teils auch selbstkritische Bewertung bot. Das Ende der Koalition kam schließlich verfrüht und unerwartet. In Wolfrums Augen agierte Rot-Grün »wie ein Fadenspinner: Viele Fäden wurden gelegt, die irgendwohin gingen, aber nicht mehr zusammengebunden wurden« (713). Erst in der Interpretation von RotGrün als Teil längerer Entwicklungslinien in der vereinigten Bundesrepublik wird deutlich, worin die leichten Kurskorrekturen bestanden und wo die Koalition tiefergehende Zäsuren vollzogen hat. Am Ende oszilliert dies zwischen dem »Unausgeschöpften « (710) und "posthumen Siegen « (711), mithin politischen Weichenstellungen, die sich viele Jahre später als richtig erwiesen haben und die auch von Kanzlerin Angela Merkel als solche anerkannt worden sind. In diesem Sinne findet sich in Rot-Grün eine Bestätigung - trotz eines turbulenten Umfeldes aus Wirtschaftskrise, internationalem Terrorismus, Globalisierung und innenpolitischem Reformdruck - bundesrepublikanischer Kontinuität und der Fähigkeit zur Selbstkorrektur politischer Maßstäbe.

Helge F. Jani 